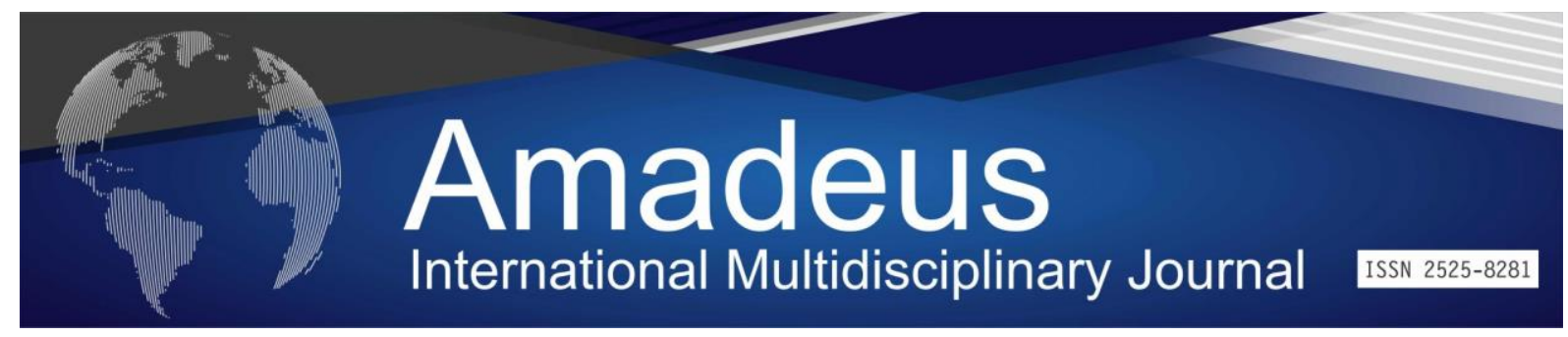

DOI: 10.14295/aimj.v5i9.142

\title{
Complications in Elderly After Proximal Femoral Fracture: an Integrating Review
}

Allex Alves Sobral de Sousal;

Carmelita Maria

Silva Sousa ${ }^{2}$;

Lucineide Coqueiro Gurgel ${ }^{3}$; Iasmin Belém Silva Queiroz ;

Francisco Rafael Soares de Sousa ;

Joanacele Gorgonho Ribeiro Nóbrega ${ }^{6}$; Marta Coêlho Bezerra Dantas ${ }^{7}$;

Aclenielle Vital Santana Duarte $^{8}$;

Dayse Christina Rodrigues Pereira Luz'; Willma José de Santana ${ }^{10}$

\begin{abstract}
Proximal femoral fractures in the elderly are mainly caused by low energy trauma, often resulting from a fall. Studies demonstrate the growing concern with the functional independence of the elderly due to the high incidence of proximal femoral fracture and its complications. The aim of this study was to identify the main factors associated with proximal femoral fractures (FFP) in the elderly and their consequences for their functional independence. The present study is defined as an integrative review. The search for scientific articles was carried out in the databases LILACS, Medline, SciElo and PubMed, from 2015 to 2019. Inclusion criteria were articles that were entirely available online and in the Portuguese, English and Spanish languages. And articles that were not available in the whole and before 2015 were excluded. In the results, it was identified that proximal femoral fractures are more frequent in the elderly with greater longevity. It has been observed that the appearance of future consequences in the elderly after FFP is often related to the long bedridden periods of this patient, impairing their functional independence. Elderly patients with FFP present a greater predisposition to reduce muscle strength, thus contributing to the appearance of future complications. These factors contribute to the morbidity and increase of the elderly mortality. FFP represent an important factor to lose functional independence in the elderly. Promote the elimination of future complications and their risk factors with therapeutic planning of individual and collective actions aimed at preventing injuries and their consequences, and thus reducing the mortality of this patient.
\end{abstract}

Keywords: Proximal femoral fracture. Elderly. Consequences. Complications.

\footnotetext{
${ }^{1}$ Master's student in Public Policy at Universidade Athenas College. Email: allexsobralfisio@ hotmail.com;

${ }^{2}$ Master's student in Public Policy at Universidade Athenas College. Email: carmelitasilva11@hotmail.com;

${ }^{3}$ Master's student in Public Policy at Universidade Athenas College. Email: lucineide.gurgel@yahoo.com.br;

${ }^{4}$ Master's student in Public Policy at Universidade Athenas College. Email: iasminbelem@ @otmail.com;

${ }^{5}$ Master's student in Public Policy at Universidade Athenas College. Email: rafa-soares_@hotmail.com;

${ }^{6}$ Master's student in Public Policy at Universidade Athenas College. Email: joanacelegorgonho@ hotmail.com;

${ }^{7}$ Master's student in Public Policy at Universidade Athenas College. Email: martha_30jpeg@ @otmail.com;

${ }^{8}$ Master's student in Public Policy at Universidade Athenas College. Email: acleniellevs@ hotmail.com;

${ }^{9}$ Post Doctoral Student in Health Sciences at the Health University Center of ABC-FMABC, Professor at the University Center of Juazeiro do Norte - UNIJUAZEIRO. Email: dayse.dcrp@ hotmail.com;

${ }^{10}$ Post Doctoral Student in Health Sciences - FMABC, PhD in Biological Sciences at UFPE and Professor at Centro Universitário de Juazeiro do Norte - UNIJUAZEIRO and Faculty of Technology - FATEC - CARIRI.

Email: wjsantana@hotmail.com.
} 


\title{
Complicações em Idosos após Fratura de Fêmur Proximal: Uma Revisão Integrativa
}

\begin{abstract}
Resumo: Resumo: As fraturas de fêmur proximal em idosos são causadas, principalmente, por trauma de baixa energia decorrentes muitas vezes de uma queda. Estudos demonstram a crescente preocupação com a independência funcional dos idosos devido elevada incidência de fratura fêmur proximal e suas complicações. Teve-se com objetivo identificar os principais fatores associados as fraturas de fêmur proximal (FFP) em idosos e suas consequências para independência funcional dos mesmos. $\mathrm{O}$ presente estudo define-se como uma revisão integrativa com abordagem qualitativa. A busca dos artigos científicos foi realizada nas bases de dados: LILACS, Medline, SciElo e PubMed. Aparte da combinação dos seguintes descritores (DeCS): "Fratura de fêmur proximal", "Idoso", "Consequências" e "Complicações". Como critérios de inclusão adotou-se artigos inteiramente disponíveis na forma on-line e nos idiomas português, inglês e espanhol. E foram excluídos artigos que não estivessem disponíveis na integra e anteriores a 2015. Nos resultados, identificou-se que as fraturas de fêmur proximal são mais frequentes nos idosos com maior longevidade. Observou-se que o aparecimento de consequências futuras em idosos após FFP está relacionado muitas vezes aos longos períodos acamado deste paciente, prejudicando sua independência funcional. Idosos acometidos por FFP apresentam maior predisposição a redução da força muscular, assim, contribuindo no aparecimento de complicações futuras, estes fatores colabora para a morbidade e aumento da mortalidade do idoso. As FFP representam importante fator para perca da independência funcional em idosos. Promover a eliminação de futuras complicações e seus fatores de risco com planejamento terapêutico de ações individuais e coletivas voltadas à prevenção de agravos e suas consequências, e assim, reduzindo a mortalidade deste paciente.
\end{abstract}

Palavras-chave: Fratura de fêmur proximal. Idosos. Consequências. Complicações.

\section{Introdução}

Fraturas de fêmur proximal (FFP) são condições comuns na população idosa e a incidência de quedas aumenta com o avançar da idade. Onde qualidade de vida durante o envelhecimento está relacionada a perda ou diminuição da capacidade funcional do idoso, que é afetada diferentemente de acordo com o sexo (Nascimento e Tavares, 2016). As mulheres idosas apresentam maior declínio das funções física, e os homens idosos apresentam maior declínio das funções relacionadas a saúde mental. O comprometimento das funções físicas e 
mentais pode ser ainda mais observadas quando o idoso sofre uma fratura de fêmur proximal (Prato et al, 2017).

Estima-se que cerca de $5 \%$ dos pacientes idosos internados com fraturas de fêmur proximal morrem durante a sua hospitalização inicial e um terço no primeiro ano após a lesão (Pimentel et al, 2015). Assim, além de indesejáveis para os pacientes, as quedas seguidas de FFP são também dispendiosas para suas famílias e para a sociedade, já que podem comprometer a independência funcional, ou seja, a capacidade do indivíduo de realizar algo com seus próprios meios, necessitando de um cuidador. Projeções futuras apontam que, no ano de 2050, seis milhões de pessoas sofrerão fratura fêmur proximal (Rodrigues et al, 2016).

Entre os fatores de risco para as quedas e fraturas destaca-se: consumo abusivo de álcool, uso de fumo, idade elevada, sexo feminino, uso de drogas psicotrópicas, incapacidade física, desequilíbrio, perda da capacidade cognitiva e presença de comorbidades, osteoporose, menopausa precoce, obesidade e sedentarismo. Dentre as fraturas, as mais comuns são as de fêmur proximal, hoje consideradas um dos maiores problemas da saúde pública, devido seu alto custo com gastos em saúde, como, a elevada incidência nas taxas de morbidade e mortalidade entre os idosos (Carneiro et al, 2016).

O envelhecimento pode ser determinado por características biológicas, influenciado por problemas de saúde e causas externas, entre elas quedas e fraturas em idosos (Classificação Internacional de Doenças CID-10). A queda é considerada um problema de saúde pública e se dá em decorrência da perda total do equilíbrio postural, mecanismos externos resultando em fratura, podendo estar relacionada à insuficiência súbita dos problemas neurais e osteoarticulares envolvidos na manutenção da postura ${ }^{6}$. Entre as principais consequências das quedas estão as fraturas e risco de morte, o medo de cair, a restrição de atividades, o aumento do risco de institucionalização e o declínio na saúde. As FFP geram, além do aumento dos custos com os cuidados de saúde, prejuízo físico e psicológico, aumento da utilização de vários serviços especializados, sobre tudo o aumento do número de hospitalizações (Freitas et al, 2015).

Reduzir as complicações das FFP nos idosos exige ações pensadas e efetivadas a partir de uma abordagem multidimensional, o que só é possível por meio da ação integrada e especializada de todos envolvidos no cuidado do idoso (Abreu e Oliveira, 2015). O modelo atual de prevenção de quedas e fraturas do Ministério da Saúde (MS) está fundamentado em três ações que estão relacionadas e integralmente associadas: construir a conscientização sobre a importância da prevenção e do tratamento das fraturas; incrementar a avaliação dos 
fatores individuais, ambientais e sociais que aumentem a probabilidade da ocorrência de novas quedas; e incentivar o planejamento e a implementação de intervenções de redução do número de fraturas, fundamentadas nas próprias experiências dos idosos-alvo (Brasil, 2017).

Assim, considerando o intenso envelhecimento populacional, a elevada incidência de quedas em idosos, contexto promissor às FFP e suas consequências para independência funcional. Assim questiona-se: Quais às consequências para recuperação da independência funcional dos idosos após fratura fêmur proximal (FFP)?

Este estudo tornou-se relevante, na medida que possibilitará o reconhecimento de aspectos relacionados às consequências da fratura fêmur proximal na população idosa, analisar os efeitos das complicações para recuperação da independência funcional do idoso e os riscos associados a redução da qualidade de vida deste paciente. Diante do exposto e da relevância do tema, o presente estudo teve como objetivo identificar os principais fatores associados as fraturas de fêmur proximal em idosos e suas consequências para independência funcional dos mesmos.

\section{Metodologia}

Utilizou-se a revisão integrativa com abordagem qualitativa, este método de revisão específica tem a finalidade de reunir e sistematizar resultados de pesquisas sobre a literatura teórica ou empírica anterior para prover o entendimento e o conhecimento do tema investigado relacionado à saúde (Muller et al, 2017). A abordagem qualitativa refere-se a uma pesquisa pela busca de informações que estejam ligadas as características inerentes ao estudo investigado, onde está sujeito ao julgamento interpretativo e subjetivo do tema referido (Taquette e Minayo, 2018).

A revisão integrativa compreende as seguintes etapas: estabelecimento do problema, ou seja, definição do tema da revisão em forma de questão ou hipótese primária; eleição da amostra após definição dos critérios de inclusão; caracterização dos estudos (definem-se as características ou informações a serem coletadas dos estudos, por meio de critérios claros, norteados por instrumento), análise dos resultados (identificando similaridades e conflitos) e apresentação e discussão dos achados (Rojas et al, 2018).

Para a construção do estudo contemplou-se as seguintes etapas: identificação do tema e definição da questão de pesquisa, estabelecimento de critérios para inclusão e exclusão dos 
estudos (amostragem), definição das categorias/informações a serem extraídas dos artigos selecionados: autor(es), ano, título, revista, idioma, objetivo(s) e conclusão.

Neste sentido, buscou-se artigos científicos nas seguintes bases de dados: LILACS (Literatura Latino-Americana e do Caribe em Ciências da Saúde), Medline (Medical Literature Analysis and Retrieval System Online), SciElo (Scientific Electronic Library Online) e PubMed (US National Library of Medicine), e a combinação dos seguintes Descritores em Ciências da Saúde (DeCS): "Fratura de fêmur proximal", "Idoso", "Consequências" e "Complicações".

Os critérios de inclusão definidos para a seleção dos artigos foram: artigos inteiramente disponíveis na forma on-line e relacionados ao objeto de pesquisa, artigos disponíveis em idiomas português, inglês e espanhol, artigos que fossem indexados nas bases de dados. Contextualizando o idoso com diagnóstico de FFP e com idade igual ou superior a 60 anos. Como critério de exclusão não se utilizou artigos que não estavam disponíveis para leitura completa em português, inglês e espanhol, não estivessem disponíveis na integra, que não estivessem disponíveis na forma gratuita on-line e artigos anteriores ao ano de 2015.

A busca foi realizada pelo acesso on-line, no período de 20 de julho a 07 agosto de 2020, e, inicialmente, foram obtidos 47 artigos. Após a leitura destes artigos, sete foram selecionados, e, posteriormente, foram incluídos outros três artigos referenciados nos estudos por atenderem aos critérios de inclusão, totalizando dez artigos que compuseram a amostra. Onde estão representados no fluxograma a seleção dos artigos incluídos na revisão. (Figura 1). 
Figura 1 - Fluxograma mostrando a seleção dos artigos incluídos na revisão.

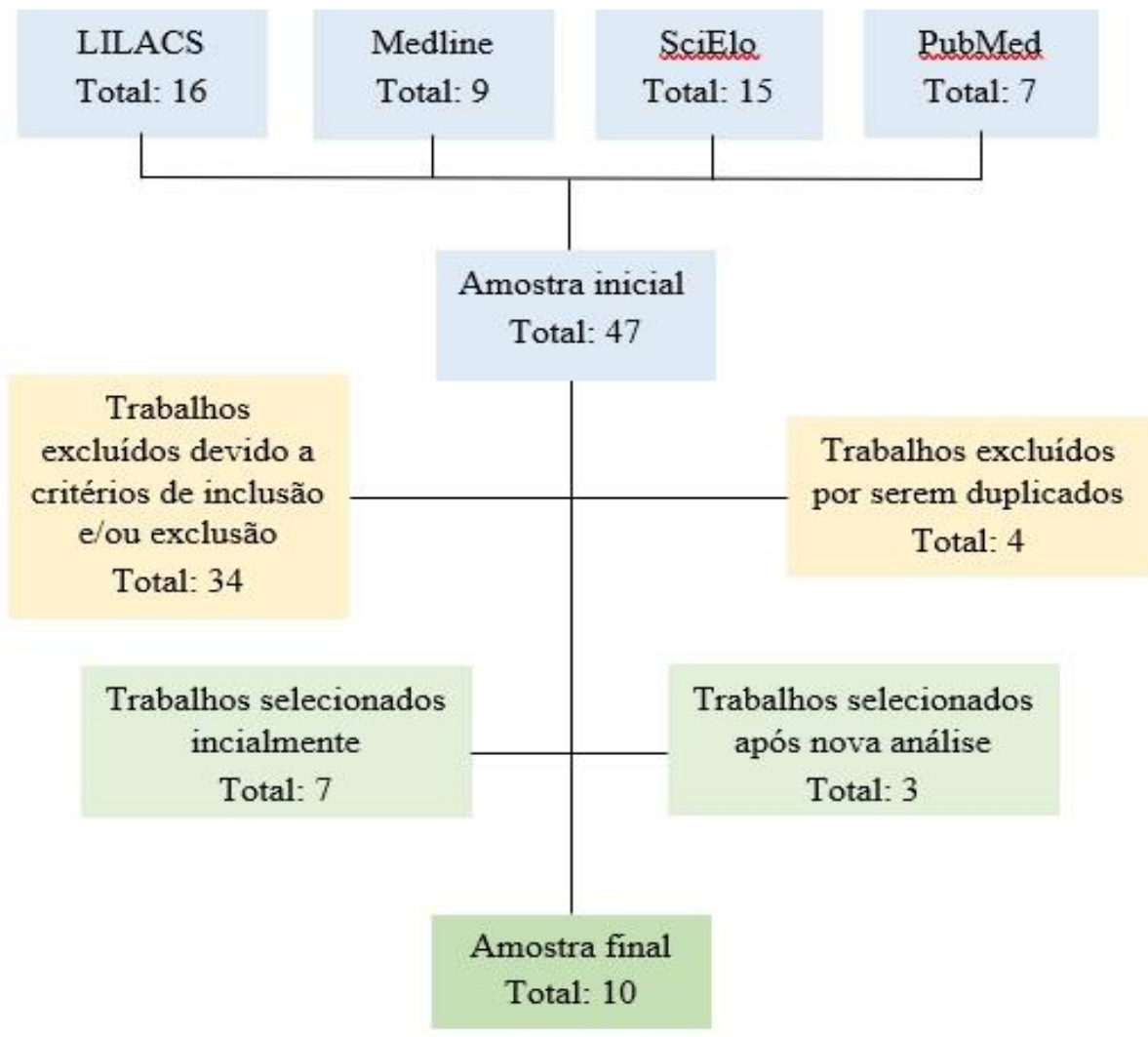

Fonte: dados da Pesquisa, 2020.

\section{Resultados e Discussão}

Com a seleção dos artigos encontrados nos quais atenderam aos critérios de inclusão estabelecidos e que tratavam sobre FFP em idosos, cujas informações retiradas estão apresentadas no (Quadro 1).

Quadro 1: Caracterização dos artigos incluídos no estudo.

\begin{tabular}{|c|c|c|c|c|c|}
\hline $\begin{array}{c}\text { AUTOR } \\
\text { (ES)/ANO }\end{array}$ & TÍTULO & REVISTA & IDIOMA & OBJETIVO(S) & CONCLUSÃO \\
\hline $\begin{array}{c}\text { Avila, et } \\
\text { al. / (2015) }\end{array}$ & $\begin{array}{c}\text { Participação } \\
\text { familiar para a } \\
\text { reabilitação de } \\
\text { idosos com } \\
\text { fratura de } \\
\text { fêmur }\end{array}$ & $\begin{array}{c}\text { Revista } \\
\text { Brasileira de } \\
\text { Enfermagem }\end{array}$ & Português & $\begin{array}{c}\text { Verificar se o modo } \\
\text { de cuidar adotado } \\
\text { pelos cuidadores } \\
\text { favoreceu a } \\
\text { recuperação da } \\
\text { independência } \\
\text { funcional dos } \\
\text { idosos após FFP. }\end{array}$ & $\begin{array}{c}\text { Recomenda-se o incentivo a } \\
\text { movimentação e a deambulação, } \\
\text { ao autocuidado e as atividades } \\
\text { de lazer, ações estas que previne } \\
\text { futuras complicações, } \\
\text { principalmente naqueles idosos } \\
\text { com maior dificuldade de } \\
\text { locomoção. }\end{array}$ \\
\hline
\end{tabular}




\begin{tabular}{|c|c|c|c|c|c|}
\hline $\begin{array}{l}\text { SOARE, et } \\
\text { al. / } \\
(2015)\end{array}$ & $\begin{array}{l}\text { Analysis of } \\
\text { factors } \\
\text { associated to } \\
\text { falls and hip } \\
\text { fractures in } \\
\text { elderly people: } \\
\text { a case-control } \\
\text { study }\end{array}$ & $\begin{array}{c}\text { Revista } \\
\text { Brasileira } \\
\text { Geriatria e } \\
\text { Gerontologia }\end{array}$ & Inglês & $\begin{array}{c}\text { Identificar os } \\
\text { principais fatores } \\
\text { associados a quedas } \\
\text { e fraturas de fêmur } \\
\text { em idosos. }\end{array}$ & $\begin{array}{l}\text { Conhecer os fatores de risco } \\
\text { para fraturas pós-queda em } \\
\text { idosos é essencial para o } \\
\text { planejamento de ações } \\
\text { individuais e coletivas voltadas } \\
\text { à prevenção deste agravo e suas } \\
\text { consequências. Atividades } \\
\text { físicas, planos terapêuticos mais } \\
\text { adequados e correção de } \\
\text { inadequações nos domicílios } \\
\text { desses indivíduos devem ser } \\
\text { orientados e incentivados }\end{array}$ \\
\hline $\begin{array}{l}\text { Sacheto e } \\
\text { Moreira } \\
(2016)\end{array}$ & $\begin{array}{l}\text { Repercussões } \\
\text { na dinâmica } \\
\text { familiar de } \\
\text { idosos com } \\
\text { fratura de } \\
\text { fêmur }\end{array}$ & $\begin{array}{c}\text { Fisioterapia } \\
\text { Brasil }\end{array}$ & Português & $\begin{array}{l}\text { Este estudo } \\
\text { analisou as } \\
\text { repercussões na } \\
\text { dinâmica familiar } \\
\text { de familiares de } \\
\text { idosos com fraturas } \\
\text { fêmur proximal de } \\
\text { (FFP) a partir de } \\
\text { suas representações } \\
\text { sociais. }\end{array}$ & $\begin{array}{c}\text { No instante que o idoso é } \\
\text { acometido por FFP, cuidados } \\
\text { familiares são necessários para } \\
\text { evitar complicações associadas } \\
\text { a fatores de risco, como, } \\
\text { aumento da incidência de novas } \\
\text { quedas e diminuição da } \\
\text { independência funcional do } \\
\text { idoso. }\end{array}$ \\
\hline $\begin{array}{c}\text { Carvalhoi } \\
\text { e Bocchii } \\
(2017)\end{array}$ & $\begin{array}{l}\text { Ancianos } \\
\text { reconociendo } \\
\text { vulnerables a } \\
\text { caídas en la } \\
\text { concreción de } \\
\text { la fractura del } \\
\text { fémur }\end{array}$ & $\begin{array}{c}\text { Revista } \\
\text { Española de } \\
\text { Enfermería }\end{array}$ & Espanhol & $\begin{array}{c}\text { Compreender a } \\
\text { experiência de } \\
\text { idosos com quedas } \\
\text { seguidas de fraturas } \\
\text { do fêmur e elaborar } \\
\text { modelo teórico } \\
\text { desse processo de } \\
\text { vivência. }\end{array}$ & $\begin{array}{c}\text { Após a fratura de fêmur } \\
\text { observa-se a necessidade da } \\
\text { implementação de programa } \\
\text { continuado de prevenção de } \\
\text { quedas, diminuindo os fatores } \\
\text { de risco para o idoso e suas } \\
\text { possíveis consequências. }\end{array}$ \\
\hline $\begin{array}{c}\text { Petros et } \\
\text { al. / } \\
(2017)\end{array}$ & $\begin{array}{l}\text { Influência das } \\
\text { fraturas do } \\
\text { fêmur } \\
\text { proximal na } \\
\text { autonomia e } \\
\text { mortalidade } \\
\text { dos pacientes } \\
\text { idosos } \\
\text { submetidos a } \\
\text { osteossíntese } \\
\text { com haste } \\
\text { cefalomedular } \\
\end{array}$ & $\begin{array}{c}\text { Revista } \\
\text { Brasileira de } \\
\text { Ortopedia e } \\
\text { Traumatologia }\end{array}$ & Português & $\begin{array}{c}\text { Determinar a } \\
\text { autonomia e a } \\
\text { mortalidade de } \\
\text { pacientes idosos } \\
\text { após FFP } \\
\text { submetidos a } \\
\text { osteossíntese do } \\
\text { fêmur com haste } \\
\text { cefalomedular. }\end{array}$ & $\begin{array}{l}\text { Eliminando-se ao máximo as } \\
\text { complicações e promovendo a } \\
\text { autonomia prévia do paciente, } \\
\text { fatores estes que influenciam } \\
\text { positivamente no resultado } \\
\text { funcional e a recuperação pós- } \\
\text { operatória. }\end{array}$ \\
\hline$\frac{\text { Davenport }}{\frac{\text { et al. / }}{(2018)}}$ & $\begin{array}{c}\text { Outcomes for } \\
\text { Elderly } \\
\text { Patients With } \\
\text { Atypical } \\
\text { Femoral } \\
\text { Fractures } \\
\text { Compared to } \\
\text { Typical } \\
\text { Femoral } \\
\text { Fractures for } \\
\text { Length of } \\
\text { Stay, } \\
\text { Discharge } \\
\text { Destination, } \\
\text { and 30-Day } \\
\text { Mortality Rate }\end{array}$ & $\begin{array}{c}\text { Geriatric } \\
\text { Orthopaedic } \\
\text { Surgery \& } \\
\text { Rehabilitation }\end{array}$ & Inglês & $\begin{array}{c}\text { Comparar se os } \\
\text { idosos que } \\
\text { apresentam fraturas } \\
\text { femorais atípicas } \\
\text { (FAAs) e os } \\
\text { fraturas femorais } \\
\text { típicas TFFs, com } \\
\text { foco na avaliação } \\
\text { da mortalidade } \\
\text { hospitalar e de } 30 \\
\text { dias. }\end{array}$ & $\begin{array}{l}\text { Com os princípios modernos de } \\
\text { atendimento ao trauma, as } \\
\text { consequências podem ser } \\
\text { minimizadas aos idosos } \\
\text { comparando os resultados } \\
\text { obtidos após os FHAs podem } \\
\text { ser equivalentes às fraturas } \\
\text { femorais típicas na população } \\
\text { geriátrica. }\end{array}$ \\
\hline
\end{tabular}




\begin{tabular}{|c|c|c|c|c|c|}
\hline $\begin{array}{l}\text { Modesto et } \\
\text { al. / } \\
(2018)\end{array}$ & $\begin{array}{l}\text { Ocorrência de } \\
\text { fratura de } \\
\text { fêmur e } \\
\text { rastreamento } \\
\text { de sinais de } \\
\text { depressão em } \\
\text { idosos }\end{array}$ & $\begin{array}{c}\text { Revista } \\
\text { Psicologia: } \\
\text { Teoria e } \\
\text { Prática }\end{array}$ & Português & $\begin{array}{l}\text { O objetivo deste } \\
\text { estudo foi } \\
\text { identificar a } \\
\text { ocorrência de } \\
\text { fratura de fêmur e } \\
\text { rastrear sinais de } \\
\text { depressão em } \\
\text { idosos. }\end{array}$ & $\begin{array}{l}\text { Concluiu-se que houve número } \\
\text { elevado de internações por } \\
\text { fratura de fêmur e sinais de } \\
\text { depressão entre os idosos, } \\
\text { sinalizando a necessidade de } \\
\text { novas investigações e ações que } \\
\text { modifiquem essa realidade, } \\
\text { impedindo complicações e } \\
\text { promovendo a sua reabilitação. }\end{array}$ \\
\hline $\begin{array}{c}\text { Pimentel et } \\
\text { al. / } \\
(2018)\end{array}$ & $\begin{array}{l}\text { Quedas entre } \\
\text { idosos } \\
\text { brasileiros } \\
\text { residentes em } \\
\text { áreas urbanas: } \\
\text { ELSI-Brasil }\end{array}$ & $\begin{array}{c}\text { Revista de } \\
\text { Saúde Pública }\end{array}$ & Português & $\begin{array}{c}\text { Determinar a } \\
\text { prevalência e os } \\
\text { fatores associados a } \\
\text { quedas em amostra } \\
\text { nacional } \\
\text { representativa da } \\
\text { população idosa } \\
\text { residente em áreas } \\
\text { urbanas. }\end{array}$ & $\begin{array}{l}\text { Os fatores associados às quedas } \\
\text { entre idosos são } \\
\text { multidimensionais, incluindo } \\
\text { características individuais e o } \\
\text { ambiente urbano, o que indica a } \\
\text { necessidade de ações intra e } \\
\text { intersetoriais para a prevenção } \\
\text { de quedas, fator este de maior } \\
\text { relevância para possíveis } \\
\text { complicações nessa população. }\end{array}$ \\
\hline $\begin{array}{c}\text { Madeiras } \\
\text { et al. / } \\
(2019)\end{array}$ & $\begin{array}{l}\text { Determinantes } \\
\text { socioeconômic } \\
\text { os e } \\
\text { demográficos } \\
\text { na assistência } \\
\text { à fratura de } \\
\text { fêmur em } \\
\text { idosos }\end{array}$ & $\frac{\text { Ciência \& }}{\underline{\text { Saúde }}}$ & Português & $\begin{array}{l}\text { O objetivo do } \\
\text { estudo foi analisar o } \\
\text { perfil da assistência } \\
\text { à fratura de fêmur } \\
\text { em idosos, } \\
\text { relacionando às } \\
\text { condições so- } \\
\text { cioeconômicas e } \\
\text { demográficas, no } \\
\text { Paraná entre os } \\
\text { anos } 2008 \text { a } 2013 \text {. }\end{array}$ & $\begin{array}{l}\text { Conclui-se que o desempenho } \\
\text { da economia exerce } \\
\text { significativa influência na } \\
\text { assistência, limitando a presença } \\
\text { de possível consequências em } \\
\text { idosos com fratura de fêmur. } \\
\text { Em relação à eficiência do } \\
\text { tratamento das fraturas de } \\
\text { fêmur, há indicativos de que a } \\
\text { presença de tratamento precoce } \\
\text { diminui as taxas de mortalidade } \\
\text { em idosos longevos exercendo } \\
\text { influência sobre o fator. }\end{array}$ \\
\hline $\begin{array}{l}\text { Zamora- } \\
\text { Navas e } \\
\text { Esteban- } \\
\text { Peña } \\
\text { (2019) }\end{array}$ & $\begin{array}{l}\text { Seasonality in } \\
\text { the incidence } \\
\text { and mortality } \\
\text { of femoral } \\
\text { fracture }\end{array}$ & $\begin{array}{c}\text { Revista } \\
\text { Americana de } \\
\text { Cirurgia } \\
\text { Ortopédica e } \\
\text { Traumatológic } \\
\text { a }\end{array}$ & Inglês & $\begin{array}{l}\text { Determinar se } \\
\text { existe uma relação } \\
\text { sazonal na } \\
\text { incidência e } \\
\text { mortalidade intra- } \\
\text { hospitalar de } \\
\text { pacientes com } \\
\text { fratura de fêmur }\end{array}$ & $\begin{array}{c}\text { A distribuição sazonal } \\
\text { de fraturas de fêmur apresentou } \\
\text { um aumento em relação à média } \\
\text { no mês de outubro e uma queda } \\
\text { em fevereiro. A mortalidade } \\
\text { aumentou em relação à média } \\
\text { no mês de agosto e diminuiu em } \\
\text { março. Devido a complicações } \\
\text { estabelecidas nestes períodos } \\
\text { aos pacientes }\end{array}$ \\
\hline
\end{tabular}

Fonte: dados da Pesquisa, 2020.

As fraturas de fêmur proximal têm repercussão seríssima na terceira idade, evidenciando um alto nível morbilidade e mortalidade, com consequências traumáticas, considerando o maior problema para recuperação da independência funcional do idosos mais longevos. Tendo maior efeito sobre o lar e seus familiares que assumem muitas vezes o papel de cuidador neste momento de maior fragilidade do idoso (Loures et al, 2015).

Contudo, medidas como alterações no ambiente domiciliar podem ser tomadas, fazendo parte das orientações aos idosos com objetivo de se evitar futuras consequências, 
como, escorregões, tropeções e assim quedas posteriores. Neste sentido, retirada de tapetes e instalação de corrimãos nas rampas, eliminação de pisos escorregadios, escadas e banheiros adaptados são medidas preventivas simples e eficientes (Daniachi et al, 2015).

Estudos demonstram um aumento significativo da incidência de FFP nas últimas décadas, principalmente, devido ao aumento da população geriátrica, visto que sua incidência é progressiva com o avançar da idade (Guerra et al, 2017). Afetando os diversos sistemas do organismo que controlam o equilíbrio, a mobilidade, a marcha e a locomoção, a idade avançada aumenta o risco de quedas e suas consequências nas pessoas idosas (Caberlon e Bos, 2015). Os fatores que reconhecidamente afetam a recuperação trazendo consequências após FFP são: idade, estado de saúde pré-fratura, estado emocional pós-cirurgia, fatores relacionados a quedas posteriores, capacidade funcional basal, disponibilidade de apoio social recebido e déficits cognitivos (Rosso et al, 2016).

As principais consequências encontradas nos idosos extremamente vulneráveis e afetados por FFP estão relacionados à história de quedas posteriores (medo de cair), uso de medicamentos, artrite, depressão, sedentarismo, deterioração na mobilidade e na marcha, deficiência nutricional, danos cognitivos e visual (Franco et al, 2016). Revelando consequências graves após o trauma para a vida do idoso relacionados aos fatores, como, desnutrição, diminuição da acuidade visual e dos reflexos, diminuição das atividades da vida diária, fragilidade óssea e, principalmente, sarcopenia. Estes fatores precisam ser atenuados evitando-se possíveis consequências aos idosos com a implementação de programas para prevenções de quedas (Gomez et al, 2017).

As complicações de maior impacto para a vida do idoso após FFP na sua grande maioria dos casos são ocasionadas por quedas posteriores. Assim, medidas preventivas que evitem suas consequências necessitam ser adotadas para que sua ocorrência seja reduzida (Litwin et al, 2018). A prática de exercícios físicos regulares pós-operatória de FFP é defendida por vários autores com objetivo de melhorar a autonomia deste paciente, melhorando sua postura e o equilíbrio corporal, melhora da marcha, além da promoção do fortalecimento e aumentar a massa muscular desses indivíduos (Camões et al, 2016).

Além de fatores prejudiciais ao sistema musculoesquelético como a marcha e equilíbrio, também foram identificadas outras consequências de características clinicas, como arritmia e insuficiência cardíaca, problemas respiratórios, bem como acidente vascular encefálico e depressão no ambiente hospitalar (Costa e Gonçalves, 2015). Estudo epidemiológico realizado com idosos após FFP em hospital-escola da região central do Rio de Janeiro demonstrou que, 
dos 167 idosos que sofreram quedas, 39 tiveram fratura de fêmur proximal. Apresentando fratura associadas às doenças crônicas (especialmente insuficiência cardíaca) e contribui para uma taxa de 8,2\% de mortalidade pós-operatória antes da alta hospitalar (Kernkamp et al, 2016).

Considerando a saúde mental, idosos com diagnóstico ou relato de depressão acometidos por FFP e que fazer uso de medicamentos antidepressivos estiveram mais propensos a quedas, um dos motivos para isto seria o fato que pessoas depressivas apresentam um maior isolamento social e serem menos ativos fisicamente, o que favorece a diminuição do equilíbrio, reduz a força muscular dos membros inferiores e do corpo. Fatores esses são determinantes para eventos de novas quedas em idosos residentes nas áreas urbanas com diagnostico de depressão (Bretanha et al, 2015).

Devido ao declínio provocado pelo envelhecimento as áreas urbanas apresentam desafios a população idosa onde encontram muitas vezes um ambiente propicio a ocorrência de quedas. Entre a maior propensão às FFP e uma nova queda estão os eventos de atravessar a rua, calçadas com níveis diferentes e degraus com tamanhos irregulares. Estes eventos são particularmente relevantes, uma vez que eles podem ter como desfechos a restrição das atividades de vida diária, fraturas e novas hospitalizações, medo de cair novamente, proporcionando o aumento do risco para incapacidades e mortalidade, assim, aumentando os custos com saúde, retardando o tratamento e a recuperação (Rocha et al, 2016).

Diversos estudos associam fatores econômicos e tratamento adequado como sendo aspectos essenciais a melhor recuperação das fraturas em idosos. Em estudo realizado com 316 pacientes que foram atendidos e tratados preventivamente com FFP, tiveram um índice de alta hospitalar precoce $(26,6 \%)$ e redução da mortalidade intra-hospitalar (17,8\%) (Stolnicki e Oliveira, 2016). Esses dados colaboram com a pesquisa bibliográfica onde determina que o tratamento preventivo pós-operatório apresentou diminuição da taxa de $23,15 \%$ de mortalidade no primeiro ano entre os idosos com fratura de fêmur (Oliveira et al, 2015).

Muitos idosos com histórico de FFP apresentam maiores chances de sofrer novas quedas. Estas em sua maioria, podem ser prevenidas e evitadas, diminuindo as consequências, elas podem gerar redução ou perda da autonomia, da qualidade de vida, da independência, aumentando do gasto financeiros e dos danos sociais, mentais e emocionais, levando à institucionalização, hospitalização e aumento da morbidade e mortalidade (Oliveira e Barba, 2017). 


\section{Considerações Finais}

Este estudo possibilitou melhor caracterização e entendimento dos fatores associados a fratura fêmur proximal e suas complicações na recuperação do idoso, estas informações são fundamentais para garantia do planejamento de ações individuais e coletivas voltadas à prevenção desses agravos e suas consequências nestes pacientes.

Considerando a melhoria do quadro de FFP, pois existe uma diminuição funcional, um isolamento social, que pode levar a uma depressão e também um aumento da morbidade e mortalidade precoce nos idosos. Contudo, torna-se necessárias ações educativas para prevenção e promoção da saúde dos idosos, visando a identificação precoce dos fatores de risco e intervindo quando necessário.

Portanto, a prevenção e a eliminação de possíveis consequências exercem um papel importante na reabilitação do idoso após a FFP, pois existe a necessidade de se elaborar planos terapêuticos mais apropriados, assim incentivando a eliminação de inadequações nos domicílios desses indivíduos são medidas que deverão ser orientadas individualmente aos pacientes, bem como disseminadas na forma de ações direcionadas à população idosa.

\section{Referências}

Abreu E. L \& Oliveira M. H. A. (2015). Avaliação da qualidade de vida dos pacientes submetidos à hemiartroplastia do quadril. Revista Brasileira de Ortopedia, São Paulo, v. 50, n. 5, p. 530-536. DOI: http://dx.doi.org/10.1016/j.rbo.2014.09.008.

Avila M. A. G. et al. (2015). Participação familiar para a reabilitação de idosos com fratura de fêmur. Revista Brasileira de Enfermagem, v. 68, n. 5, p. 601-607. DOI: http://dx.doi.org/10.1590/0034-7167.2015680517i.

Brasil. (2017). Ministério da Saúde (BR), secretaria de atenção à saúde, departamento de ações programáticas estratégicas. Caderneta de saúde da pessoa idosa. 4.ed. Brasília.

Bretanha A. F. et al. (2015). Sintomas depressivos em idosos residentes em áreas de abrangência das unidades básicas de saúde da zona urbana de Bagé, RS. Revista Brasileira de Epidemiologia, v. 18, n. $1, \quad$ p. $1-12 . \quad$ DOI: http://dx.doi.org/10.1590/19805497201500010001.

Briggs R. et al. (2018). Does baseline depression increase the risk of unexplained and accidental falls in a cohort of community dwelling older people? Internacional Journal of Geriatric Psychiatry, v. 33, n. 2, p. 205-211. DOI: https://dx.doi.org/10.1002/gps.4770. 
Caberlon I. C. \& Bos A. J. G. (2015). Diferenças sazonais de quedas e fraturas em idosos gaúchos. Ciência \& Saúde Coletiva, Rio de Janeiro, v. 20, n. 12, p. 3743-3752. DOI: http://dx.doi.org/10.1590/1413-812320152012.20602014.

Camões M. et al. (2016). Exercício físico e qualidade de vida em idosos: diferentes contextos sócio comportamentais. Motricidade, v. 12, n. 1, p. 96-105. DOI: http://dx.doi.org/10.6063/motricidade.6301.

Carneiro J. A. et al. (2016). Quedas em idosos não institucionalizados no norte de Minas Gerais: prevalência e fatores associados. Revista Brasileira de Geriatria e Gerontologia, Rio de Janeiro, v. 19, n. 4, p. 613-25. DOI: https://dx.doi.org/10.1590/180998232016019.150110 .

Carvalho C. J. A \& Bocchi S. C. M. (2017). Ancianos reconociendo vulnerables a caídas en la concreción de la fractura del fémur. Revista Española de Enfermería, v. 70, n. 2, p. 279286. DOI: http://dx.doi.org/10.1590/0034-7167-2016-0392.

Costa J. L. \& Gonçalves D. M. (2015). A extrema vulnerabilidade na transição dos cuidados hospitalares para o domicílio: Uma análise sobre determinantes sociais de saúde. Lusíada. Intervenção Social, Lisboa, v. 42, n. 45, p. 175-189.

Daniachi D. et al. (2015). Honda EK. Epidemiologia das fraturas do terço proximal do fêmur em pacientes idosos. Revista Brasileira de Ortopedia, São Paulo, v. 50, n. 4, p. 371-377. DOI: http://dx.doi.org/10.1016/j.rbo.2014.07.014.

Davenport D. et al. (2018). Outcomes for elderly patients with atypical femoral fractures compared to typical femoral fractures for length of stay, discharge destination, and 30-day mortality rate. Geriatric Orthopaedic Surgery \& Rehabilitation, v. 9, n. 1, p. 1-4. DOI: http://dx.doi.org/10.1177/2151459318820222.

Franco L. G. et al. (2016). Fatores associados à mortalidade em idosos hospitalizados por fraturas de fêmur. Revista Brasileira de Ortopedia, São Paulo, v. 51, n. 5, p. 509-14. DOI: http://dx.doi.org/10.1016/j.rbo.2015.10.009.

Freitas M. G. et al. (2015). Idosos atendidos em serviços de urgência no Brasil: um estudo para vítimas de quedas e de acidentes de transito. Ciência \& Saúde Coletiva, Rio de Janeiro, v. 20, n. 3, p. 701-712. DOI: https://dx.doi.org/10.1590/1413-81232015203.19582014.

Gomez F. et al. (2017). A simple algorithm to predict falls in primary care patients aged 65 to 74 years: the international mobility in aging study. JAMDA - Journal America Medicine $\begin{array}{lllllll}\text { Direct Association, } & \text { v. } 18, \quad \text { n. } & 9, & \text { p. } & 774-779, & \text { DOI: }\end{array}$ https://dx.doi.org/10.1016/j.jamda.2017.03.021.

Guerra M. T. E. et al. (2017). Mortalidade em um ano de pacientes idosos com fratura do quadril tratados cirurgicamente num hospital do sul do Brasil. Revista Brasileira de Ortopedia, São Paulo, v. 52, n. 1, p. 17-23. DOI: http://dx.doi.org/10.1016/j.rbo.2016.04.005.

Litwin H. et al. (2018). The complex association between fear of falling and mobility limitation in relation to late-life falls: a share-based analysis. Journal of Aging and Health, v. 30, n. 6, p. 987-1008. DOI: https://dx.doi.org/10.1177/0898264317704096. 
Loures F. B. et al. (2015). Custo-efetividade do tratamento cirúrgico da fratura do quadril em idosos no Brasil. Revista Brasileira de Ortopedia, São Paulo, v. 50, n. 1, p. 38-42. DOI: http://dx.doi.org/10.1016/j.rbo.2014.04.002.

Kernkamp C. L. et al. (2016). Perfil de morbidade e gastos hospitalares com idosos no Paraná, Brasil, entre 2008 e 2012. Caderno de Saúde Pública, v. 32, n. 7, p. 1-14. DOI: http://dx.doi.org/10.1590/0102-311x00044115.

Madeiras J. G. et al. (2019). Determinantes socioeconômicos e demográficos na assistência à fratura de fêmur em idosos. Ciência \& Saúde Coletiva, v. 24, n. 1, p. 97-104. DOI: http://dx.doi.org/10.1590/1413-81232018241.03862017.

Modestor R. F. et al. (2018). Ocorrência de fratura de fêmur e rastreamento de sinais de depressão em idosos. Revista Psicologia: Teoria e Prática, São Paulo, v. 20, n. 2, p. 325338. DOI: http://dx.doi.org/10.11606/s1518-8787.2018052000103.

Muller M. G. et al. (2017). Uma revisão da literatura acerca da implementação da metodologia interativa de ensino peer instruction (1991 a 2015). Revista Brasileira de Ensino de Física, v. 39, n. 3, p. 3403-3405. DOI: http://dx.doi.org/10.1590/1806-9126-rbef2017-0012.

Nascimento J. S. \& Tavares D. M. S. (2016). Prevalência e fatores associados a quedas em idosos. Texto \& Contexto - Enfermagem, v. 25, n. 2, p. 1-9. DOI: https://dx.doi.org/10.1590/0104-07072016000360015.

Oliveira C. C. \& Borba V. C. Z. (2017). Epidemiologia das fraturas de fêmur em idosos e seu custo para o estado do Paraná, Brasil. Associação Ortopédica Brasileira, v. 25, n. 4, p. 155158. DOI: http://dx.doi.org/10.1590/1413-785220172504168827.

Oliveira T. C. et al. (2015). Diferenciais sócio-demográficos da mortalidade de idosos em idades precoces e longevas. Revista Baiana de Saúde Pública, v. 39, n. 2, p. 249-61. DOI: http://dx.doi.org/10.5327/z0100-0233-2015390200006.

Petros R. S. B. et al. (2017). Influência das fraturas do fêmur proximal na autonomia e mortalidade dos pacientes idosos submetidos a osteossíntese com haste cefalomedular. Revista Brasileira de Ortopedia, São Paulo, v. 52, n. 1, p. 57-62. DOI: http://dx.doi.org/10.1016/j.rbo.2017.06.011.

Pimentel W. R. T. et al. (2015). Quedas e qualidade de vida: associação com aspectos emocionais em idosos comunitários. Geriatria e Gerontologia Aging, v. 9, n. 2, p. 42-48. DOI: http://dx.doi.org/10.5327/z24472115201500020002.

Pimentel W. R. T. et al. (2018). Quedas entre idosos brasileiros residentes em áreas urbanas: ELSI-Brasil. Revista de Saúde Pública, v. 52, n. 2, p. 12-21. DOI: http://dx.doi.org/10.11606/s1518-8787.2018052000635.

Prato S. C. F. et al. (2017). Frequência e fatores associados a quedas em adultos com 55 anos e mais. Revista de Saúde Pública, v. 51, n. 37. DOI: https://dx.doi.org/10.1590/s15188787.2017051005409. 
Rocha A. S. et al. (2016). Influência do cuidador informal na reabilitação do idoso em pósoperatório de fratura de fêmur proximal. Revista Gaúcha de Enfermagem, v. 37, n. 1, p. 145-59. DOI: http://dx.doi.org/10.1590/19831447.2016.01.51069.

Rodrigues G. D. et al. (2016). Prevenção de quedas no idoso: revisão da literatura brasileira. Revista Brasileira de Prescrição e Fisiologia do Exercício, v. 10, n. 59, p. 431-437. DOI: http://dx.doi.org/10.1590/s141381232008000400023.

Rojas J. et al. (2018). Aspectos pedagógicos na educação em enfermagem: Revisão Integrativa. Investigación y Educación Enfermería, v. 36, n. $3 . \quad$ DOI: http://dx.doi.org/10.17533/udea.iee.v36n3e03.

Rosso F. et al. (2016). Prognostic factors for mortality after hip fracture: operation within 48 hours is mandatory. Injury, v. 47, n. 4, p. 91-97. DOI: https://doi.org/10.1016/j.injury.2016.07.055.

Sacheto R. M \& Moreira M. A. S. P. (2016). Repercussões na dinâmica familiar de idosos com fratura de fêmur. Fisioterapia Brasil, v. 17, n. 6, p. 534-543. DOI: http://dx.doi.org/10.33233/fb.v17i6.694.

Soares D. S. et al. (2015). Analysis of factors associated to falls and hip fractures in elderly people: a case-control study. Revista Brasileira Geriatria Gerontologia, Rio de Janeiro, v. 18, n. 2, p. 239-248. DOI: http://dx.doi.org/10.1590/1809-9823.2015.14022.

Stolnicki B. \& Oliveira L. G. (2016). Para que a primeira fratura seja a última. Revista Brasileira de Ortopedia, São Paulo, v. 51, n. 2, p. 121-6. DOI: http://dx.doi.org/10.1016/j.rbo.2015.03.009.

Taquette R. S. \& Minayo M. C. (2016). Análise de estudos qualitativos conduzidos. Physis: Revista de Saúde Coletiva, Rio de Janeiro, v. 26, n. 2, p. 417-434. DOI: http://dx.doi.org/10.1590/s0103-73312016000200005.

Zamora-Navas P. \& Esteban-Peña M. (2019). Seasonality in the incidence and mortality of femoral fracture. Revista Americana de Cirurgia Ortopédica e Traumatológica, v. 67, n. 4, p. 56-66. DOI: http://dx.doi.org/10.1136/ip.6.1.16.

How to cite this article (APA format):

Sousa, Allex Alves Sobral de; Sousa, Carmelita Maria Silva; Gurgel, Lucineide Coqueiro; Queiroz, Iasmin Belém Silva; Sousa, Francisco Rafael Soares de; Nóbrega, Joanacele Gorgonho Ribeiro; Dantas, Marta Coêlho Bezerra; Duarte, Aclenielle Vital Santana; Luz, Dayse Christina Rodrigues Pereira; Santana, Willma José de (2020). Complications in elderly after proximal femoral fracture: an integrating review. Am. In. Mult. J., Jul to Oct. (9) 5, 238-251.

Received: 09/21/2020;

Accepted: 10/17/2020. 